\title{
A framework for solving the configuration problem in Wireless Sensor Networks
}

\author{
A. Gogu, D. Nace and Y. Challal \\ Laboratoire Heudiasyc UMR CNRS 6599 \\ Université de Technologie de Compiègne \\ 60205 Compiègne Cedex, France \\ \{ada.gogu, dnace, ychallal\}@ @ds.utc.fr
}

\begin{abstract}
Wireless Sensor Network Configuration Problem seeks to dimension a WSN while taking into account both the traffic pattern, data aggregation and the transmission range assignment. Regarding the objectives of the problem, we first intend to minimize the overall energy consumption of the network and next to guarantee node energy consumption fairness between the sensors. Previously, we discussed this problem for the linear network. Herein, we consider a twodimensional network where sensors are uniformly placed in the target area. The network is divided into cells and the Network configuration problem has to determine the number of cells and their respective sizes such that the above objectives are met. A framework, based on the dynamic programming algorithm, is proposed to solve this problem. This framework provides optimal solution, is scalable and has a low computational complexity.
\end{abstract}

Index Terms-WSN, Dynamic programming, Network configuration;

\section{INTRODUCTION}

A typical Wireless Sensor Network (WSN) is composed of a set of tiny motes that sense their environment and transmit sensed information, hop-by-hop, to a processing workstation through the Base Station (BS). Nowadays, WSN represents an emerging disruptive technology with potentially a wide range spectrum of applications. We refer the reader to [11, 2] for a summary of recent applications. Faced with stringent constraints affecting, for example, energy, bandwidth and memory use, WSN technology needs to be carefully managed in order to meet the requirements of applications. Optimization techniques and strategies are applied at the physical, access control, network and application layers to improve its performance. A primary concern in wireless sensor networks is the energy constraint. A carefully designed network can be a very effective mean to conserve energy and therefore extend the lifetime of the network.

In our previous work [3], we were focused on the network configuration problem for the linear network case. We observed that $31 \%$ of energy could be saved if the network is divided according to the method proposed in [3]. In this paper, we will consider the network configuration problem in WSN for the two dimensional network. We assume that the sensors are placed in the target area and send periodically their data to the sink. As the sink may be located far from the nodes, these ones have to relay their data to other sensor nodes according to a multihop transmission scheme. As remarked in previous work [8, 6, 7], this data gathering scheme leads to uneven energy depletion of the sensors. Hence, the sensors next to the BS spend more energy because they have to relay also the data of the other sensors. The imbalance energy depletion gets a huge importance in sensor networks as it can cause the death of the whole network. To remedy this problem, different approaches are proposed which deal with load balancing, re-routing, deployment, etc. In our case, we consider the network configuration problem which can be managed in the planning phase of network design. More specifically, the problem assumes a uniform node distribution and a circular network area. This area is divided into concentric circles forming coronas. Nodes belonging to a corona will forward the data generated by the nodes themselves together with data generated by higher-level coronas. Each node in a given zone adjusts his transmission range according to sizes of the zone to whom it belongs. Besides the transmission range assignment, node energy is also affected by the amount of data that it relays. For a given node, the amount of data that it relays is not depended only on its respective position in the target area but also on the data aggregation demands which are mostly related to the type of application. Therefore, we take the aggregation into account in our model.

Solving this problem is not trivial. For a network configuration scheme we have to find the optimal number of coronas and zones and the length of each corona according to the aggregation model in order to i) minimize the total energy consumption and ii) guarantee fair energy depletion for all the nodes. We propose a dynamic programming framework for this problem. It solves optimally the problem and can be adapted for different types of aggregation schemes, energy models estimation and different objectives. Let us first briefly recall the principle of Dynamic Programming (DP). DP is a sequential approach, proposed by Bellman [1] for optimizing a given objective function. The problem is thus broken down into stages and the aim at every stage is to select the optimal decision so that the objective is optimized over the current number of stages. Hence, in each stage we solve only once the corresponding subproblem. The results of each stage are stored and later used to backtrack the optimal values. The most typical 
example of a DP implementation is the shortest path. If we agree that $(A \ldots B \ldots C)$ is the shortest path between the points $\mathrm{A}$ and $\mathrm{C}$ and passes through $\mathrm{B}$, then $\mathrm{AB}$ is evidently the shortest path between the points $\mathrm{A}$ and $\mathrm{B}$.

The remainder of the paper is organized as follows. Our literature review is presented in section III Section III introduces the assumptions a of the problem and the notations used throughout the work. In section IV we introduce our algorithm which intend to minimize the total energy consumption and next, in $\nabla$ we focus on the strategy which guarantees the energy fairness expenditure. Section VIprovides comparative computational tests for several scenarios. Finally, section VII concludes the paper.

\section{RELATED WORKS}

In order to mitigate the energy holes in a data gathering network, [9] dialed with the network configuration problem seen in terms of general design guidelines for the WSN. They considered a network where sensors are uniformly deployed and the data are fully aggregated in each hop. The problem required to divide the network into coronas and to determine the optimal number of coronas and their respective sizes while taking into account only the transmission energy. In order to minimize the total energy consumption, the authors showed that the coronas' width should the same. Next, they propose an iterative process which determines the corona's width for achieving energy expenditure balancing.

The network configuration problem is discussed also in [14], which proposes a method for dividing the network into coronas, then the coronas into subcoronas and finally the subcoronas into zones such that the load in every zone is balanced. In this model, there is always a mapping between the sensors of a given zone belonging to a corona, and the sensors of another zone, belonging to an upstream corona. The problem of finding the optimal number of coronas is modeled as an optimization problem and a simulated annealing algorithm is proposed. The problem of dividing the coronas into subcoronas and zones is solved iteratively. This network division scheme assumes that the coronas and the subcoronas have the same width and that the number of zones is the same for each of them.

In [10], on the other hand, the number of coronas and their respective lengths are assumed to be known, and the transmission range must be assigned for each corona so as to maximize network lifetime. The transmission range will actually determine the next hop corona. The algorithm proposed for this problem builds a graph in which the nodes represent the coronas and the edges represent the feasible wireless communication links between any two coronas. The algorithm builds a set of spanning trees for each node, and those having the best performances in terms of network lifetime are selected. Among the spanning trees that include the most distant corona and the $B S$, the one with the longest lifetime is retained as the solution to the problem.

In [13] the transmission structure is given by a particular matrix called the routing model and the network data throughput is assumed to be a constant. The question is then to determine the percentage of total data that each corona will forward and generate. The problem is easier in the case where this matrix is independent of density: here densities are iteratively calculated from the top down for each corona (the number of coronas and their respective widths are assumed to be known). The other scenario, where the routing decision depends on the node density, is also solved iteratively. The [13] strategy starts out from the assumption that densities are known, computes the routing matrix, calculates the densities like for the first scenario and repeats the process from the beginning until the load is balanced between the coronas. In [12], on the other hand, data collection is modeled through a Poisson process. The density control algorithm then uses a logic almost identical to [13]. This method provides a non-uniform random deployment in which the density increases closer to the $B S$.

In this paper, we provide a more general approach for the network configuration problem which can consider data aggregation, different energies (for e.x. receiving and idle energy) regarding two different objectives. Moreover, it provides optimal solution for the problem.

\section{PROBLEM DESCRIPTION}

We assume that the sensors are uniformly distributed in a circular monitoring area. There is only one sink, positioned at the center of the zone. The sensors will transmit their information according to a many-to-one traffic pattern using a multihop scheme. Total energy consumption will be minimized by optimally dividing the area into concentric coronas $C_{1}, C_{2}, \ldots C_{n}$ centered at the sink and then subdividing each corona into a given number of zones. Fig. 1 gives an example of network division with 3 coronas containing respectively 1,4 and 8 zones. Moreover, the length of every corona can be varied. For each zone there is only one sensor, the cluster head, that is able to receive information from other sensors in the zone, aggregate this information and transmit it to the downstream zone closer to the $B S$. We assume that any sensor in the zone is a potential cluster head. Notice that we can easily shift from this model to a specific-sensor placement model in which the cluster head position must be explicitly determined by adapting the radio transmission range allocated to each zone.

For the above problem, we use an aggregation model proposed in [8], which has the form $y=m x+c$ where $x$ and $y$ are the node input and output information quantities respectively, $m$ varies in $[0,1]$ and $c$ is a constant. Moreover, we assume that each node will generate an unit traffic.

Finally, for the network model as described above, the questions to be answered are:

- What is the optimal number of coronas that minimizes energy across the whole network? What are their respective radii?

- What is the optimal number of zones that a corona is divided in? 


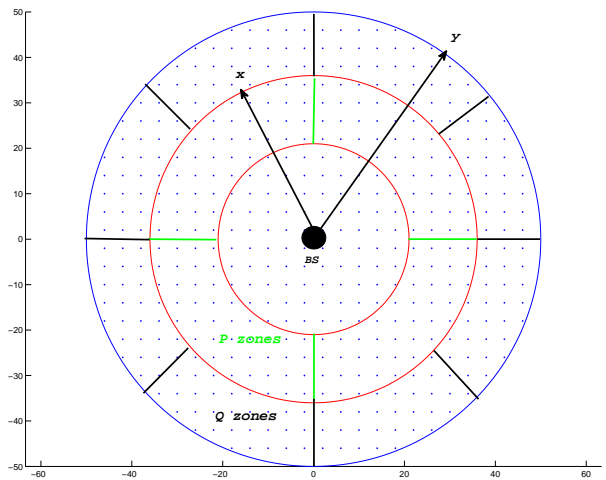

Figure 1: Two-dimensional network

Here we assume that each zone has a cluster head that will aggregate the traffic and transmit it towards the cluster head of the downstream zone closer to the $B S$.

The energy model that we use to estimate the energy consumed by them is proposed in [4]. $E_{T X}$ denotes the energy used for transmitting and $E_{R X}$ the energy used for receiving, as in equation (1).

$$
\left\{\begin{array}{l}
E_{T X}=\left(E_{\text {elec }}+E_{a m p} d^{\gamma}\right) \cdot B \\
E_{R X}=E_{r e c} \cdot B
\end{array}\right.
$$

In these equation, $d$ corresponds to the distance of communication. Besides the energy spent for receiving and transmitting the data, sensors consume energy also in the idle mode. For the sake of the simplicity, the idle energy is not considered for this problem.

In order to simplify the further reading of the paper, we present below the table of notations $\square$

\section{Minimal energy COnsumption - Strategy 1}

Our solution for the network configuration problem is based in two steps i) parameter discretization of the radius and the number of zones for each corona respectively, and ii) dynamic programming implementation. We assume that the sensor network has an uniform node distribution $n_{d}$ and each sensor generates a constant traffic $\alpha \cdot \beta$. Hence, for a such network with radius $R$ and number of zones $N$, we apply Algorithm 1

In the initialization phase, the algorithm computes the energy spent in a simple cell $(N=1)$ in which all the sensors transmit their information directly to the $B S$ using the same transmission range $y$. The energy values for the first vector $E_{y, 1}^{1}$ are computed according to formula (2).

$$
E_{y, 1}^{1}=\left(E_{\text {elec }}+E_{\text {amp }} \cdot y^{\gamma}\right) \cdot n_{d} \pi y^{2} \alpha \beta
$$

In the next step, the number of coronas is two ( $n=$ $2)$ and the algorithm computes the matrix $\left\{E_{y, Q}^{2} \mid(y \leq\right.$ $R, Q \leq N)\}$. For each $n$ coronas' number, a new matrix $\left\{E_{y, Q}^{n} \mid(y \leq R, Q \leq N)\right\}$ is generated where $N$ gives the maximal number of the zone divisions of the last corona. The elements of each matrix for a given $n$ are computed based on the dynamic programming principle where the last

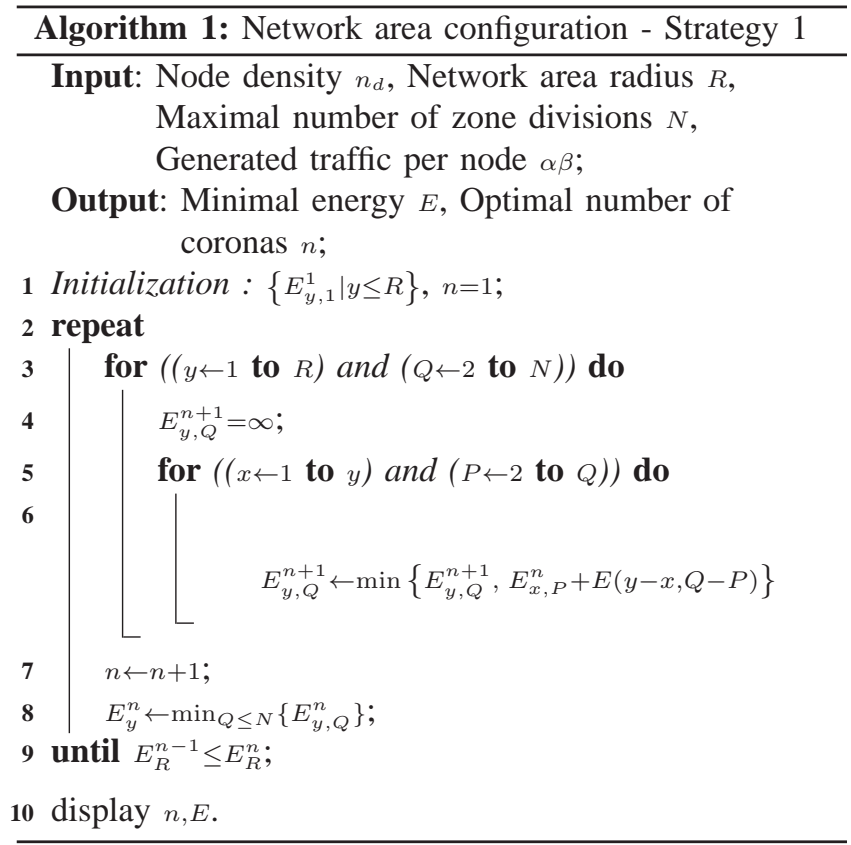

hop term is represented by $E(y-x, Q-P)$ and computed according to formula (3). Here $(y-x)$ gives the length of the last corona and Q (resp. P) gives the number of zones in the last (resp. last but one) corona. A simple illustration is given in Fig. 1

$$
E(y-x, Q-P)=Q \cdot f_{1}(y, x, P)+f_{2}(y, x, Q)
$$

where:

$$
f_{1}(y, x, P)=\left(E_{\text {elec }}+E_{a m p} \cdot\left(R_{t}(y, x, P)\right)^{\gamma}+E_{\text {rec }}\right) \cdot \alpha \beta
$$

with

$$
R_{t}(y, x, P)=\left(y^{2}+x^{2}-2 y x \cos \left(\frac{2 \pi}{P}\right)\right)^{\frac{1}{2}}
$$

Equation (4) gives the energy used by the cluster head of each zone in the $n^{t h}$ corona to transmit the information towards the next cluster head closer to the $B S$ while using a transmission range defined by the $R_{t}(y, x, P)$ function. As in the sensor placement problem, the transmission range is calculated based on the cosine formula. However, in this problem we do not know the cluster head placement, therefore we consider the maximal angle for computing the transmission range which depends only on the number of zones $P$ of the precedent level $n-1$.

$$
\begin{aligned}
f_{2}(y, x, Q)= & \left(E_{\text {elec }}+E_{a m p}\left(R_{t}(y, x, Q)\right)^{\gamma}+E_{r e c}\right) \\
& \cdot n_{d} \pi\left(y^{2}-x^{2}\right) \alpha \beta
\end{aligned}
$$

Equation (6) gives the energy consumed by all the sensors in a corona for performing intra-zone transmissions 1 . Regarding algorithm 1 we calculate the matrix $\left\{E_{y, Q}^{n} \mid(y \leq\right.$ $R, Q \leq N)\}$ for subsequent values of parameter $n$. Next, we find the $E_{R}^{n}$ which is the minimal value of the last row of the matrix $\left\{E_{R, N}^{n}\right\}$. We stop the calculations when we reach some $n$ such that $E_{R}^{n} \leq E_{R}^{n+1}$. During the

\footnotetext{
${ }^{1}$ Intra-zone transmissions include the receiving and transmitting operations inside a zone between the cluster head and the other sensors in the zone.
} 
execution of the algorithm we keep track of those values $\left(x_{i}, P_{i}\right)$ that allow to achieve the minimum of energy at the current iteration. The solution can then easily be built by backtracking these stored values. At the end of the algorithm we obtain the optimal number of coronas for minimum energy consumption, as well as the number of zones for each intermediate corona and the corresponding radius.

a) Remark: In the general case, where the aggregation model is $y=m x+c$ the equation in the line 6 of the Algorithm 1 is modified according to the equation (7).

$$
\begin{aligned}
E_{y, Q}^{n+1} \leftarrow & \min \left\{E_{y, Q}^{n+1}, E_{x, P}^{n}+m \cdot\left(\left(E_{\text {elec }}+E_{a m p} x^{2}+E_{r e c}\right)\right.\right. \\
& \left.\cdot \pi\left(y^{2}-x^{2}\right) \alpha \beta+E(y-x, Q-P)\right\}
\end{aligned}
$$

Next, the equation (3) will be modified as in 8

$$
E(y-x, Q-P)=Q \cdot f_{1}(y, x, P)\left(y^{2}-x^{2}\right) \cdot+f_{2}(y, x, Q)
$$

For the simulations, the nodes are uniformly deployed in the circular area and generate a constant amount of traffic. The cluster head of each zone aggregates all the information that it receives and transmits a constant amount of traffic to the closest cluster head. Table II lists the system configuration parameters in detail whereas the values of the energy model are given in Table I] For these parameters, some experimental results for Algorithm 1 are presented in Table III] As we can observe, the optimal number of coronas is 9, and the algorithm determines the radius of each corona and

\begin{tabular}{|c|c|c|}
\hline Type & Parameter & Value \\
\hline \multirow{3}{*}{ Network } & Area radius & $50 m-300 m$ \\
\hline & Node distribution & Uniformly \\
\hline & Node density & 0.02 \\
\hline \multirow{4}{*}{ Application } & $\|$ Data generation rate $\beta$ & $100 \mathrm{bps}$ \\
\hline & $\|$ Data traffic per node $\alpha$ & 0.003 Erlang \\
\hline & $\epsilon$ & 0.07 \\
\hline & Compression ratio & $m=0 \mid c=\alpha \cdot \beta$ \\
\hline
\end{tabular}
the number of zones, both of which decrease uniformly.

Table II: Simulation parameters

The energy consumptions of the whole network for different network radii and node densities are presented in Fig. 2.

\section{ENERGY FAIRNESS}

Assuring energy fairness between the network clusters is an alternative objective to minimizing total energy consumption. Here the optimal solution to the problem gives a network configuration such that differences in the energy consumed by the sensors of different cells and the overall

\begin{tabular}{|c|c|c|}
\hline \multirow{2}{*}{ Cell sizes } & \multicolumn{2}{|c|}{ Algorithm results } \\
\hline & $\begin{array}{c}\text { Radius of each } \\
\text { corona from the } B S\end{array}$ & Number of zones \\
\hline corona 9 & 100 & 27 \\
\hline corona 8 & 90 & 24 \\
\hline corona 7 & 80 & 21 \\
\hline corona 6 & 70 & 18 \\
\hline corona 5 & 60 & 15 \\
\hline corona 4 & 50 & 12 \\
\hline corona 3 & 40 & 9 \\
\hline corona 2 & 30 & 6 \\
\hline corona 1 & 22 & 1 \\
\hline
\end{tabular}
energy consumed are minimized. To solve this problem, we propose the Algorithm 2 Here $E_{y, Q}^{n}$ represents the total
Table III: Optimal configuration for overall energy consumption, network radius $R=100 \mathrm{~m}$

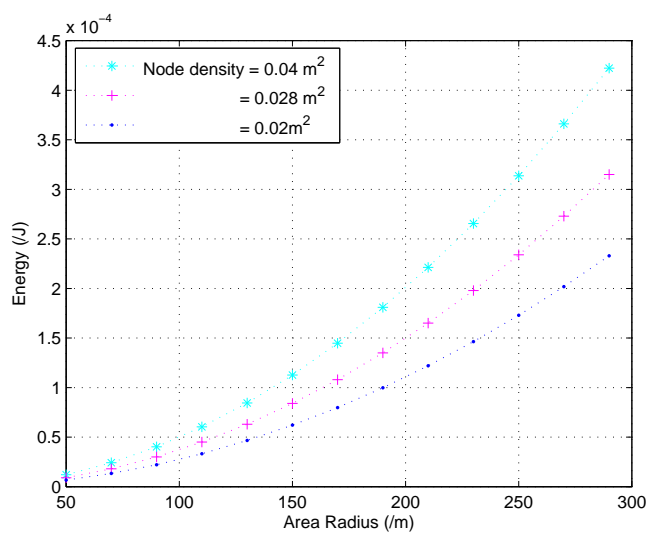

Figure 2: Energy versus network area radius

energy consumption while $\frac{E_{y, Q}^{n}}{N 1}$ is the average energy consumed by a sensor when the network's radii is $y$ and its $n^{\text {th }}$ corona is divided into $Q$ zones. The term $N 1$ is the number of the sensors deployed in the area with a radii $y$. In the initialization phase, matrix $E_{y, 1}^{1}$ is computed according to equation (2). The $E(y-x, Q-P), f_{1}(y, x, P), R_{t}(y, x, P)$ and $f_{2}(y, x, Q)$ functions are given by the corresponding equations (3), (4), (5) and (6). The ratio $\frac{f_{1}(y, x, P)+f_{2}(y, x, Q)}{N 2}$ gives the sensor's energy in the respective zone while $N 2=\frac{\left(y^{2}-x^{2}\right) \cdot 2 \pi \cdot n_{d}}{Q}$ is the corresponding number of the sensors in this zone.

At first, the algorithm finds the network division $\left(x_{i}, P i\right.$ values) for a given $n$ which guarantees a fairness consumption of energy for each sensor in the network with a coefficient $\epsilon$. Second, by iterating this procedure for different $n$, it seeks the minimal value of total energy consumption. This value is reached for some $n$ which satisfies the inequality $E_{R}^{n-1} \leq E_{R}^{n}$. Algorithm 2 is implemented for the scenario described in Section III The application and parameter values are defined in Table $\amalg$ wheres the energy parameters are given in Table [. The results are shown in Table IV] For application needs, the number of zones in one corona should be a multiple of this in the downstream corona. 


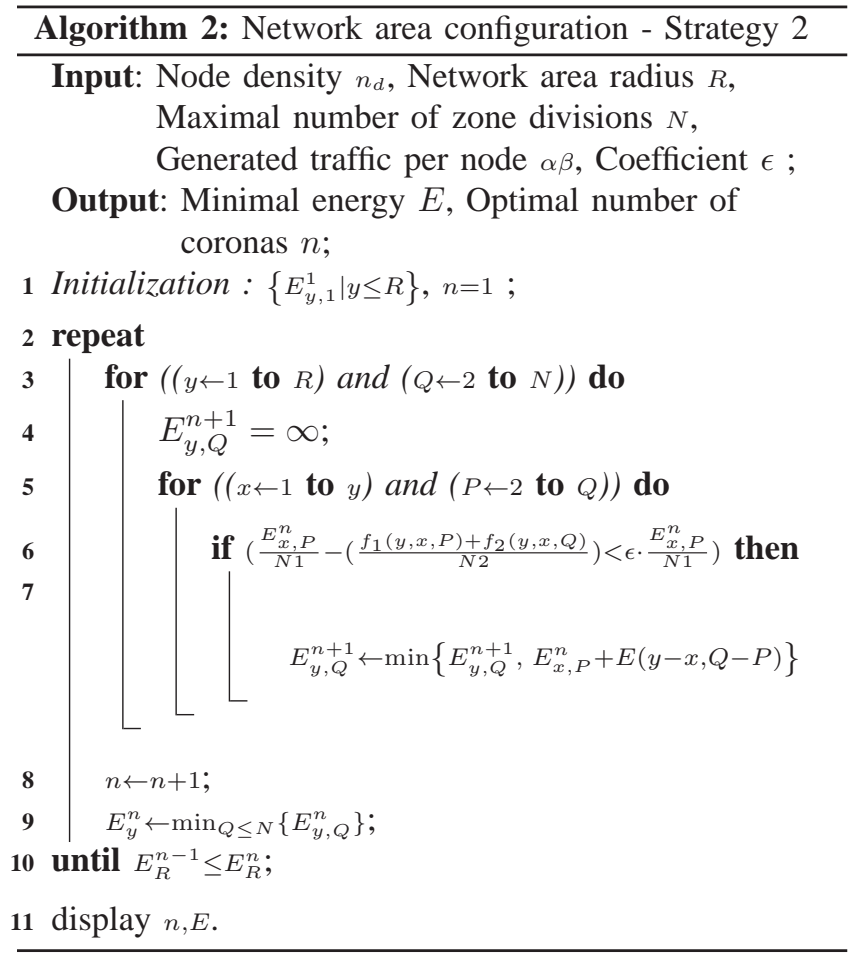

However, this can be easily taken into account by assuming that the number $Q$ of zones, in algorithm 2 , is a multiple of $P$.

\begin{tabular}{|c|c|c|}
\hline \multirow{2}{*}{ Cell sizes } & \multicolumn{2}{|c|}{ Algorithm results } \\
\hline & $\begin{array}{l}\text { Radius of each } \\
\text { corona from the } B S\end{array}$ & Number of zones \\
\hline corona 8 & 100 & 30 \\
\hline corona 7 & 86 & 27 \\
\hline corona 6 & 72 & 21 \\
\hline corona 5 & 60 & 15 \\
\hline corona 4 & 48 & 12 \\
\hline corona 3 & 36 & 9 \\
\hline corona 2 & 24 & 6 \\
\hline corona 1 & 12 & 1 \\
\hline
\end{tabular}

Table IV: Optimal configuration for energy fairness, network radius $R=100 \mathrm{~m}$

For the general case of the aggregation model, the changes proposed in IV-0a should be considered.

\section{NUMERICAL RESULTS}

Now we look at the differences between Strategy 1 and PEDAP (Power Efficient Data Gathering and Aggregation Protocol) proposed in [5]. The PEDAP algorithm builds a near optimum spanning tree and is designed for data gathering in sensor networks. One basic assumption of this algorithm is full aggregation, meaning that each node will transmit only one packet to its parent regardless of the number of received data packets. In both cases, we

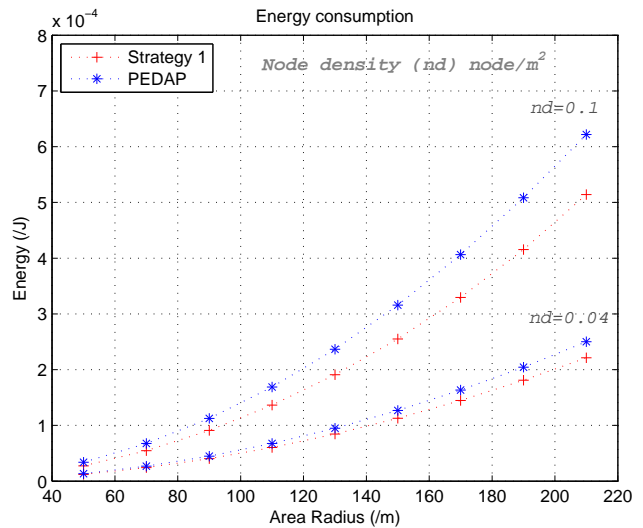

Figure 3: PEDAP and Strategy 1

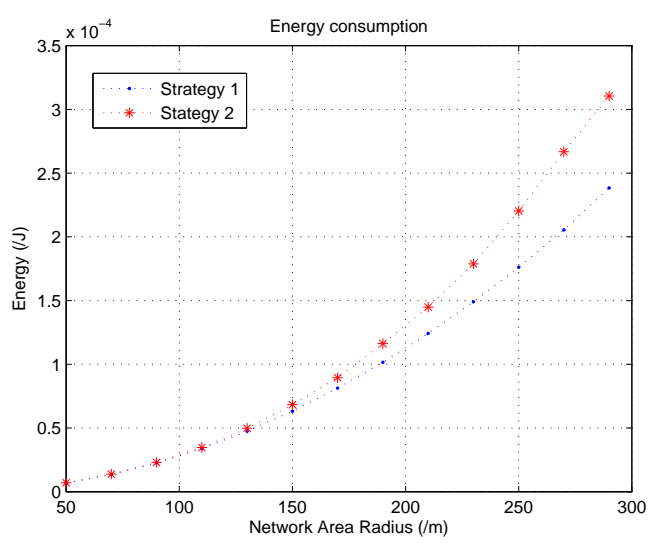

Figure 4: Energy consumption

assume that nodes are uniformly distributed in a circular area. To calculate the energy consumed by PEDAP we construct a spanning tree that includes all the nodes. In the spanning tree we differentiate between terminal and innetwork nodes. The terminal nodes use energy only for transmitting, while the others use energy for transmitting and receiving, in accordance with equations (1). In both scenarios each node has $\alpha$ Erlang of traffic, and the radio data rate is $\beta$ bps. The results of the comparison are given in Fig. 3 Our algorithm outperforms PEDAP, and more so in the case of large dense networks, which shows that it is quite scalable. The overall energy consumption for Strategies 1 and 2 is given in Fig. 4

Through simulation we also remarked that the behavior of energy function toward the node density changes is similar for both strategies.

For the network configuration problem we have looked at two strategies which seek respectively to i) minimize the energy consumption and ii) ensure a fair energy distribution while minimizing the total energy consumption. The trade-offs between the two strategies are in terms of global energy, fairness and complexity. The results show that Strategy 1 presents a lower bound of network energy consumption, but also that Strategy 2 is very close to this bound (see Fig. 4). 


\section{CONCLUSIONS}

In this paper we analyze the network configuration problem for a many-to-one WSN. For a given network where the nodes are uniformly distributed, the problem is to determine the optimal number of coronas/zones and their respective sizes such that some energy objectives are met. We show that an appropriate configuration of the WSN, addressing the coronas configuration and the respective power assignments, leads to considerable savings in energy. Our algorithms, whose aims are to minimize the total energy consumption and/or to ensure fairness in the energy consumption by different nodes, are based on the dynamic programming method. Our overall observation is that dynamic programming is an effective method for handling trade-offs between the parameters for a number of variants of the network configuration problem. It provides an optimal solution to the problem for a given objective function and reduces the computational complexity in comparison with other methods proposed in the literature. Moreover, the method can be adapted to different energy consumption models without increasing the computational complexity of the solution. In the future we shall be looking at the effects of combining different routing protocols with solutions obtained for the network configuration problem. We think that this sort of combination will be of interest at the maintenance phase and for implementation in real world applications.

\section{REFERENCES}

[1] R. Bellman (1957). Dynamic Programming. Princeton University Press, ISBN 0486428095, 2003.

[2] D. Culler, D. Estrin, and M. Srivastava. Overview of sensor networks, special issue in sensor networks. IEEE Computer, 37(8):41-49, 2004.

[3] A. Gogu, D. Nace, and Y. Challal. Energy-aware network configuration for wireless sensor networks. NBIS, pages 22-29, 2011.

[4] W. R. Heinzelman, A. P. Chandrakasan, and H.Balakrishnan. Energy efficient communication protocol for wireless microsensor networks. Proceeding of the 33rd Hawaii International Conference on System Sciences, 2000.

[5] O.T. Hüseyin and I. Körpeoğlu. Power efficient data gathering and aggregation in wireless sensor networks. SIGMOD Rec., 32:66-71, 2003.

[6] F. Ingelrest, D. Simplotryl, and I. Stojmenovic. Target transmission radius over lmst for energy-efficient broadcast protocol in ad hoc networks. In In Proceedings of the IEEE International Conference on Communications (ICC04, 2004.

[7] J. Li and P. Mohapatra. Analytical modeling and mitigation techniques for the energy hole problem in sensor networks. Pervasive and Mobile Computing, pages 233-254, 2007.

[8] V.P. Mhatre, C. Rosenberg, D. Kofman R. Mazmudar, and N. Shrof. Design guidelignes for Wireless Sensor
Networks: Communication, Clustering and Aggregation. Ad Hoc Networks, 2:45-63, 2004.

[9] S. Olariu and I. Stojmenovic: Design guidelines for maximizing lifetime and avoiding energy holes in sensor networks with uniform distribution and uniform reporting. IEEE INFOCOM, pages 1-12, 2006.

[10] C. Song, M. Liu, J. Cao, Y. Zheng, H. Gong, and G. Chen. Maximizing network lifetime based on transmission range adjustment in wireless sensor networks. Computer Communications, 32:1316-1325, 2009.

[11] Ning Xu. A survey of sensor network applications. IEEE Communications Magazine, 40, 2002.

[12] Y.Tsai, K. Yang, and S. Yeh. Non-uniform node deployment for lifetime extension in large-scale randomly distributed wireless sensor networks. AINA Proceedings, pages 517-524, 2008.

[13] Y. Yun and Y. Xia. A method for deciding node density in non-uniform deployment of wireless sensor network. http://www.cise.ufl.edu/ yx1/publication.html.

[14] H. Zhang and H. Shen. Balancing energy consumption to maximize network lifetime in data-gathering sensor networks. IEEE Trans. on Par. and Distrib. Sys., 20:1526-1539, 2009. 
Table I: Table of notations

\begin{tabular}{|c|c|c|}
\hline Type & Parameter & Description \\
\hline \multirow{11}{*}{ Network } & $d$ & Network length. \\
\hline & $R$ & Network radius. \\
\hline & $x$ & Network length from the source. \\
\hline & $k_{i}=\frac{x_{i}}{d}$ & Ratio between the sensors' distances. \\
\hline & \multirow{2}{*}{$n$} & Number of hops, deployment problem. \\
\hline & & Number of cells, network configuration problem. \\
\hline & $\gamma_{0}$ & The angle between two nodes in the uppermost circle. \\
\hline & $r$ & Ratio between the nodes in two consecutive circles. \\
\hline & $l_{i}$ & Transmission range for nodes in the $i_{t h}$ circle. \\
\hline & \multirow{2}{*}{$N_{i}$} & Number of nodes placed in the $i_{t h}$ circle, deployment problem. \\
\hline & & Number of zones in the $i_{t h}$ corona, network configuration problem. \\
\hline \multirow{7}{*}{ Application } & $\beta$ & Data generation rate in bps \\
\hline & $\alpha$ & Data traffic per node in Erlang \\
\hline & $\epsilon$ & A very small constant \\
\hline & $m$ & Compression ratio \\
\hline & $c$ & Coefficient $c=\alpha \cdot \beta$ \\
\hline & $f_{n}(d)$ & $\begin{array}{l}\text { The total energy function for a network with a length (d) and } n \\
\text { hops. }\end{array}$ \\
\hline & $\delta y=1$ & Distance discretion \\
\hline \multirow{9}{*}{ Energy } & $E_{\text {elec }}$ & Energybit consumed by the transmitter electronics. \\
\hline & $E_{a m p}$ & Energybit consumed by the amplifier. \\
\hline & $E_{r e c}$ & Energybit consumption of the receiving circuitry \\
\hline & $E_{\text {idle }}$ & Energy consumption during the idle mode. \\
\hline & $B$ & Number of bits \\
\hline & $T x_{\max }$ & Maximal transmission range \\
\hline & $\gamma$ & Path loss exponent $[2-6]$ \\
\hline & $L=\left\{l_{1}, l_{2}, \cdots l_{m}\right\}$ & Discrete distance transmission of a sensor \\
\hline & $E=\left\{e_{1}, e_{2}, \cdots e_{m}\right\}$ & Discrete energy transmission of a sensor \\
\hline
\end{tabular}

